\title{
Large area image sensing structures based on a-SiC:H: a dynamic characterization
}

\author{
M. Fernandes ${ }^{\mathrm{a}, *}$, M. Vieira ${ }^{\mathrm{a}}$, I. Rodrigues $^{\mathrm{a}}$, R. Martins ${ }^{\mathrm{b}}$ \\ a ISEL, Electronics Telecommunications and Computer Department, R. Conselheiro Emídio Navarro, P 1949-014 Lisboa, Portugal \\ ${ }^{\mathrm{b}}$ FCT-UNL Materials Science Department, Campus da FCT-UNL, Quinta da torre, 2825 Monte da Caparica, Portugal
}

Received 10 June 2003; received in revised form 17 February 2004; accepted 1 March 2004

Available online 2 July 2004

\begin{abstract}
In recent works large area hydrogenated amorphous silicon $\mathrm{p}-\mathrm{i}-\mathrm{n}$ structures with low conductivity doped layers were proposed as single element image sensors. The working principle of this type of sensor is based on the modulation, by the local illumination conditions, of the photocurrent generated by a light beam scanning the active area of the device. In order to evaluate the sensor capabilities is necessary to perform a response time characterization. This work focuses on the transient response of such sensor and on the influence of the carbon contents of the doped layers. In order to evaluate the response time a set of devices with different percentage of carbon incorporation in the doped layers is analyzed by measuring the scanner-induced photocurrent under different bias conditions.
\end{abstract}

(C) 2004 Elsevier B.V. All rights reserved.

Keywords: Spectral response; Image sensor; Large area; Heterostructures

\section{Introduction}

Large area hydrogenated amorphous silicon $\mathrm{p}-\mathrm{i}-\mathrm{n}$ structures with low conductivity doped layers were proposed as single element image sensors [1,2]. The laser scanned photodiode (LSP) is fundamentally different from the other electrically scanned image sensors since it is based on one single sensing element with an opto-mechanical readout system. The image to acquire is optically mapped on to the photosensitive surface and a low-power light spot scans the sensor in the raster mode, the portion of the photocurrent generated by the moving light spot is recorded as the image signal, which magnitude depend from the local image intensity.

The physical process of the laser scanning image acquisition is explained by the modulation of the electrical field across the junction under non-uniform steady-state illumination [3]. This information can be extracted when a weak light spot is scanning the device; in the dark regions the carriers generated by the probe beam are separated by the junction electric field and collected. Those generated inside the illuminated regions can diffuse or drift in the lateral direction. A large number of those carriers will, however, re-

\footnotetext{
* Corresponding author. Tel.: +351-21-831-7289; fax: +351-21-831-7114.

E-mail address: mfernandes@isel.pt (M. Fernandes).
}

combine inside the amorphous bulk (low ac value of the photocurrent).

Several enhancements on the LSP sensor were proposed [3] resulting in an optimization of the sensor structure. Recent works were devoted to the optimization of the readout parameters, like scanner beam spot size, image acquisition time, and sensor bias [4].

While some was carried out on the transient response of $\mathrm{p}-\mathrm{i}-\mathrm{n}$ structures $[5,6]$, this work intends to evaluate the dependence of the dynamic response of the sensor on the layers composition and electrical bias.

\section{Sensor structure}

The sensors used in this work are large area $(4 \mathrm{~cm} \times 4 \mathrm{~cm})$ amorphous $\mathrm{p}-\mathrm{i}-\mathrm{n}$ structures in the assembly glass $/ \mathrm{ZnO}: \mathrm{Al} / \mathrm{p}$ $(\mathrm{Si}: \mathrm{H}) / \mathrm{i}(\mathrm{Si}: \mathrm{H}) / \mathrm{n}(\mathrm{Si}: \mathrm{H}) / \mathrm{Al}$. The semiconductor layers were fabricated by plasma enhanced chemical vapor deposition, at $13.56 \mathrm{MHz}$ radio frequency [7]. The deposition pressure was $27 \mathrm{~Pa}$, the substrate temperature was held at $110^{\circ} \mathrm{C}$ and the rf-power was $4 \mathrm{~W}$. Low doping levels were used during the $\mathrm{n}$ and $\mathrm{p}$ layers deposition. The front transparent contact ( $\mathrm{ZnO}: \mathrm{Al}$ ) was produced by rf-sputtering and the metal back contact (Al) by thermal evaporation.

In this work three structures with different materials in the doped layers, as seen in Fig. 1, are analyzed: sam- 


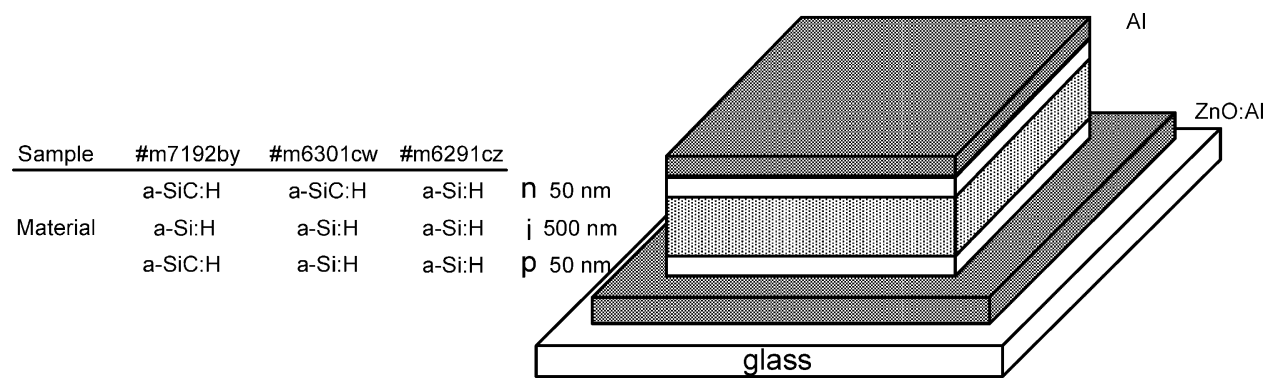

Fig. 1. Sensor structure.

ple \#m6291cz is a homojunction; sample \#m6301cw has carbon incorporated in the n-type doped layer and sample \#m7192by was fabricated with carbon in both doped layers.

The structures were characterized by spectral response, capacitance-voltage, and current-voltage measurement in dark and under different optical bias conditions as described elsewhere [8].

\section{Measurement technique}

One way to extract information about local illumination conditions is based on the use of a light probe, which scans the active area of the device. The measured photocurrent will be the sum of two components, one due to the image itself and another from the probe beam (scanner). In order to extract the image intensity information on each position, we have to separate the two components of the photocurrent. Two processes can be used to accomplish this task: synchronous, or ac amplification. In the first the probe beam is modulated at a fixed frequency and the signal is recovered by the use of a lock-in amplifier. This method is very useful for low scanning speeds due to its sensitivity and noise rejection, but cannot be used for fast scanning. The second one is based on the use of a simple current to voltage converter with low input impedance.

The scanner-induced photocurrent was measured using either a lock-in amplifier or a current to voltage converter. In both cases all the measurements were performed with a scanner wavelength of $633 \mathrm{~nm}$ (He-Ne laser). The scanning and acquisition process is controlled by a microcomputer which stores the current as a two dimensional array of discrete values, $I_{\mathrm{m}, \mathrm{n}}$, each one representing the photocurrent induced by the scanner light at the selected position. An optical system enables the change of the image and scanner beam intensity and wavelength.

\section{Results}

\subsection{Response to pulsed light}

When the chopped beam method is used for photocurrent separation, it is important to know how the signal changes with the modulation frequency. For these measurements a mechanical chopper modulated the light with a maximum frequency of $4 \mathrm{kHz}$. The sensor was illuminated with a red light pattern and the photocurrent due to the modulated beam was measured, with the beam positioned in dark and illuminated zones, using a lock-in amplifier.

As seen in Fig. 2 the signal in both regions is maximum for a frequency close to $1 \mathrm{kHz}$ and rapidly decreases for frequencies higher than $2 \mathrm{kHz}$. If we subtract both signals, we end up with the illuminated to dark ratio, which continuously decreases as the frequency increases. A tradeoff between signal amplitude and light to dark signal ratio exists for frequencies lower than $2 \mathrm{kHz}$, for higher values both the signal and difference decreases which limits the sensor performance.

The lock-in amplification gives the best results for low scan speeds due to its intrinsic noise immunity characteristics and sensitivity, together with the rejection of the current generated by the image, which is not modulated.

\subsection{Line scanning frequency}

When a fast scanning speed is necessary, the lock-in amplifier cannot be used because of the limitation in the modulation frequency presented in the previous section. In this case a low noise current to voltage converter is used and the separation between the currents due to the image and to the scanner beam is performed after by a simple subtraction. With this method, the overall intensity of the image

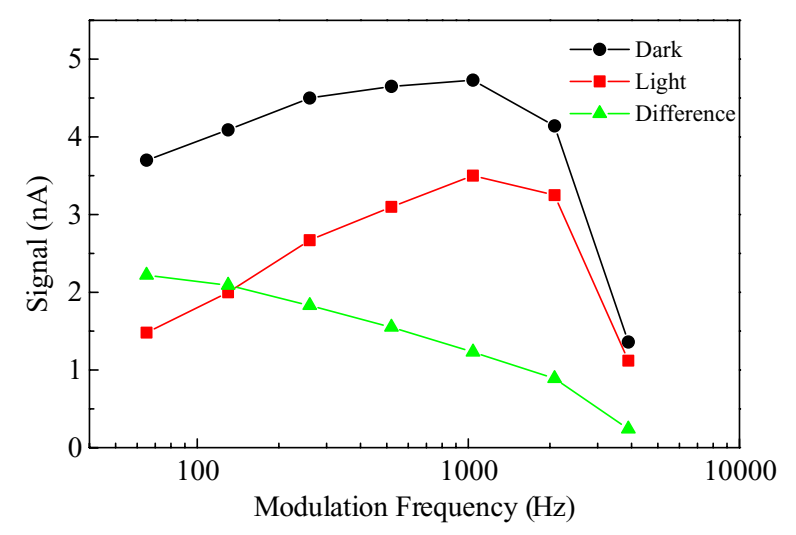

Fig. 2. Sensor response to modulated light. 

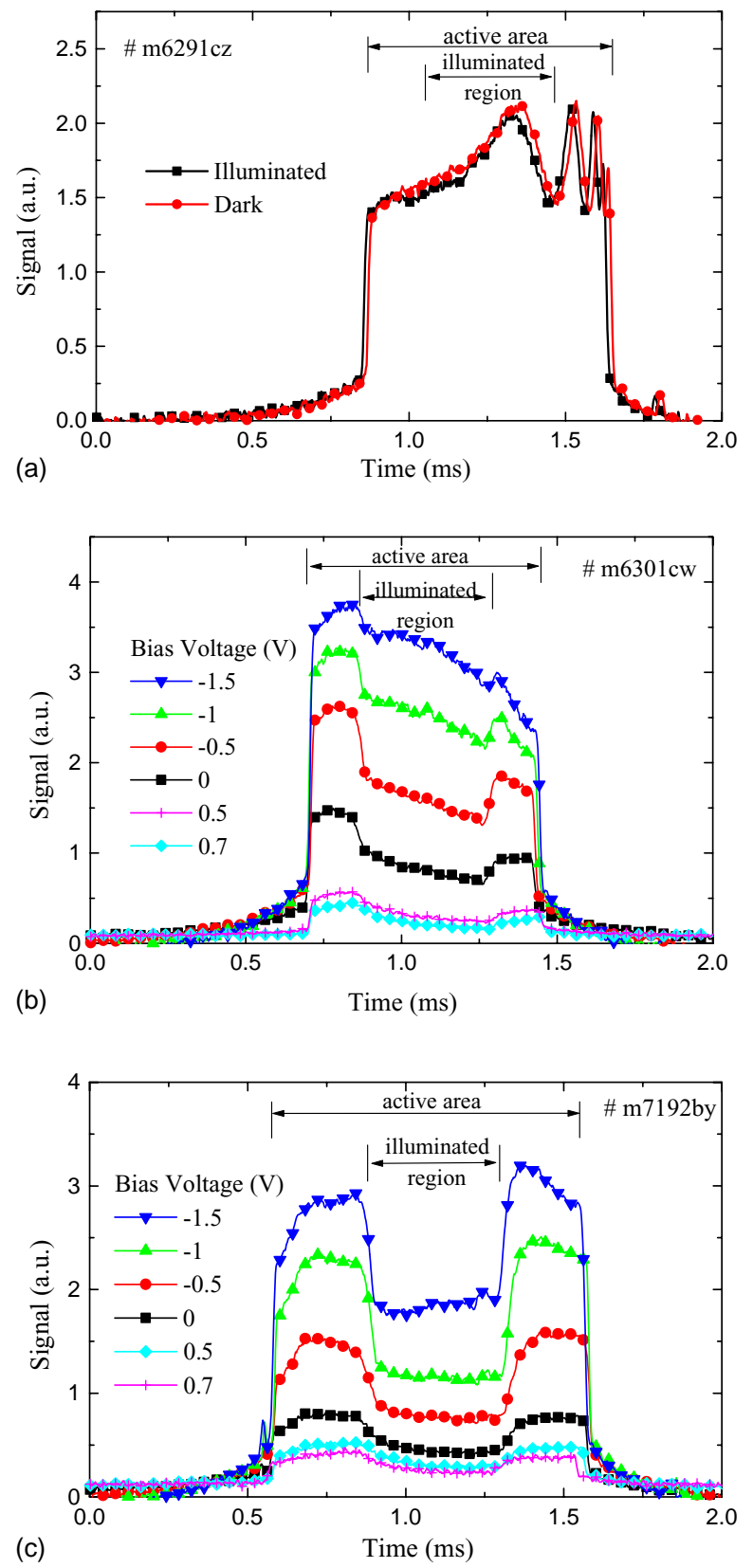

Fig. 3. Single line scans under different bias voltages for: (a) an homojunction; (b) a-SiC:H based n-type layer; and (c) a-SiC:H based $\mathrm{n}$ and $\mathrm{p}$ layers.

has to be controlled in order to avoid the saturation of the amplifier.

Fig. 3 shows the result of single line scans under different electrical bias and for the three structures when the central region of the active area of the sensors was illuminated with red light $(633 \mathrm{~nm})$, as depicted in the pictures. The scanning speed was kept constant in all the sensors, but their active area size and relative position was different which is evident in the figures. Fig. 3a presents two scans, in dark and under illumination, for the homojunction, showing that the scanner-induced photocurrent is not influenced by the local

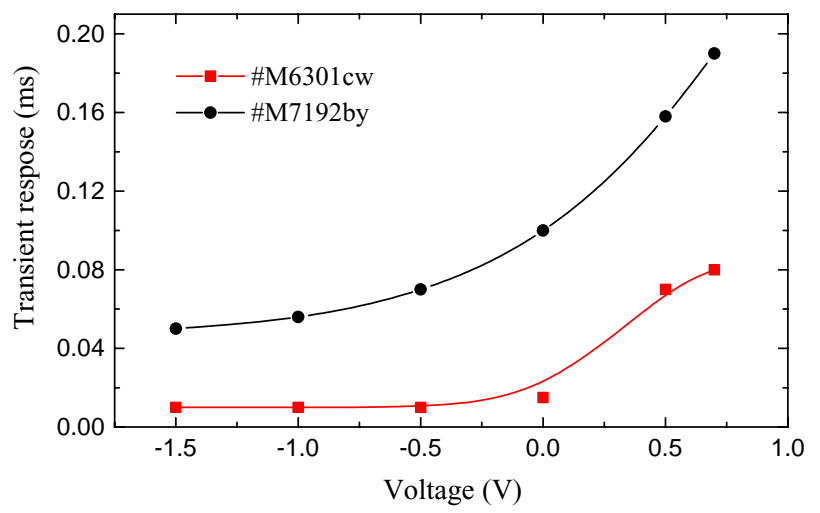

Fig. 4. Image signal, difference between dark and illuminated region scanner induced photocurrents, for the two heterostructures.

illumination conditions. The variation in the signal observed across the active area is due to the lack of uniformity in the layers, this effect is also observed in the other structures and gives origin to a fixed pattern noise which can be avoided by subtracting the signal acquired in a scan performed in dark.

If a higher band gap and lower conductivity material is used in the n-layer (Fig. 3b) the scanner-induced photocurrent becomes dependent on the local illumination conditions, which enables the use of the structure as an image sensor. The decrease in the signal in the illuminated regions can be ascribed to a localized decrease in the charge carrier collection due to the spatial modulation of the internal electrical field, caused by non-uniform illumination. One can observe also a variation in the sensitivity of the sensor (relation between signal in dark and illuminated regions) with the bias voltage, which becomes clearer in Fig. 4.

The structure with carbon incorporated in both doped layers (Fig. 3c) presents higher image sensitivity and a different dependence on the bias voltage. In both heterostructures is observed an increase in the signal with reverse bias that can be explained by the increase of the collection efficiency caused by a higher internal electrical field. The opposite happens under forward bias where the signal drops to zero.

Fig. 4 shows the signal difference between dark and illuminated regions as a function of voltage for the two heterostructures, the homojunction is not represented since this difference would be zero. The image sensitivity is highly influenced by the composition of the doped layers. When both layers have carbon incorporated, leading to a higher band gap and a higher resistivity, the image sensitivity is higher and its maximum is shifted towards higher values of reverse voltage. This is happens as a consequence of the different internal electrical field distribution in the two structures.

\subsection{Transient response}

Fig. 5 represents the transient response of samples \#m7192by and \#m6301cw, the plot shows the time necessary for the signal to decrease to $10 \%$ of its maximum when the scanning beam crosses from a dark to an illuminated 


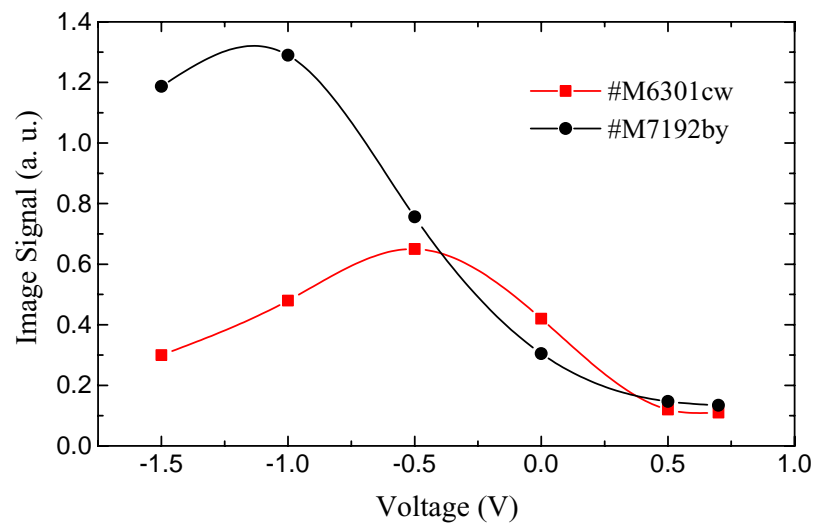

Fig. 5. Transient response of the two heterostructures.

region. Both curves have the same trend but they differ in the values. The response time is influenced the sensor internal capacitance and resistance, which are dependent on the illumination conditions, and by the readout circuitry impedance. The later was kept constant at $10 \mathrm{k} \Omega$ on all the measurements.

When the sensor is reverse biased its overall capacitance decreases and the response time lowers accordingly, both curves follow this trend in some extent. The curve for sample \#m6301cw shows a constant value for reverse bias, but this is a consequence of the process used to measure its value, since factors like lateral currents on the doped layers and the steepness of the transition from dark to illuminated regions.

The difference in the absolute values between the two structures is due to their series resistance which is higher in sample \#m7192by due to the higher resistivity of both doped layers.

\section{Conclusions}

The dynamic characteristics of a simple $\mathrm{p}-\mathrm{i}-\mathrm{n}$ diode used as an image sensor was evaluated and the results explained with basis in the internal physical process. Results show that depending on the readout technique a high sensitivity (lock-in) or a high speed (current-voltage converter) can be achieved.

Results show that the scanner induced photocurrent has a maximum for chopping frequencies around $1 \mathrm{kHz}$, while the image response time is expected to be less than $100 \mu \mathrm{s}$, depending on the bias voltage and on the composition of the doped layers.

As conclusion the dynamic properties of the sensor show that the image acquisition can be performed at frame rates close to the usually used for commercial image acquisition applications, a frame rate of $10 \mathrm{fps}$ was achieved for $100 \times 100$ pixels readout without a significant degradation in the image quality. Recent work [9] showed that controlling the voltage bias, color separation is achieved, further investigation of the sensor performance as to be done in or- der to characterize the influence of the image wavelength on the dynamic behavior.

\section{Acknowledgements}

This work has been financially supported by projects Praxis/P/EEI/12183/1998 and POCTI/ESE/38689/2001.

\section{References}

[1] M. Vieira, M. Fernandes, J. Martins, P. Louro, A. Maçarico, R. Schwarz, M. Schubert, IEEE Sens. J. 1 (2) (2001) 158-167.

[2] M. Vieira, M. Fernandes, J. Martins, P. Louro, A. Maçarico, R. Schwarz, M. Schubert, Mater. Res. Soc. Symp. Proc., San Francisco, USA, 2000.

[3] P. Louro, Y. Vygranenko, R. Schwarz, M. Fernandes, M. Vieira, J. Gloeckner, M. Schubert, Amorphous and heterogeneous silicon-based films-2001, Mater. Res. Soc. Symp. Proc., San Francisco, USA, 664 (2001).

[4] M. Fernandes, Y. Vygranenko, M. Vieira, Appl. Surf. Sci. 184 (1-4) (2001) 408-412.

[5] R. Bruggemann, C. Main, G.H. Bauer, J. Non-Cryst. Solids 164-166 (1993) 663-666.

[6] R.A. Street, J. Non-Cryst. Solids 164-166 (1993) 643-652.

[7] C. Koch, M. Ito, M. Schubert, J.H. Werner, Mater. Res. Soc. Symp. Proc. 575 (1999) 749.

[8] P. Louro, M. Vieira, Y. Vygranenko, M. Fernandes, R. Schwarz, M. Schubert, Appl. Surf. Sci. 184 (2001) 144-149.

[9] M. Vieira, M. Fernandes, A. Fantoni, P. Louro, R. Schwarz, Mater. Res. Soc. Symp. Proc., San Francisco, USA, 2002.

\section{Biographies}

Miguel Fernandes was born in Portugal in 1970. In 1993, he became Researcher in the Center of Excellence for Microelectronics and Optoelectronics Processes-UNINOVA, Lisbon, Portugal. He graduated in Physics and Materials Engineering by the Faculty of Sciences and Technology from the New University of Lisbon in 1995. Currently he is Assistant Professor in the Electronics Department of ISEL, Lisbon, Portugal and investigator in the Group of Applied Research in Microelectronic Optoelectronic and Sensors-GIAMOS in the same institution.

Manuela Vieira was born in Lisbon, Portugal in 1951. She graduated in Physics by the Faculty of Science of the University of Lisbon in 1974. In 1986, received the Master of Science in Solid State Physics-Microelectronics by the New University of Lisbon. At that time she became Auxiliary Professor of Semiconductor and Microelectronics in ISEL, Lisbon where she is now Coordinator Professor in Semiconductor. In 1993, she received the $\mathrm{PhD}$ in Semiconductor Materials from the New University of Lisbon. Currently she is the Head of the Group in Applied Research in Microelectronic Optoelectronic and Sensors-GIAMOS/ISEL in Lisbon, Portugal. Dr. M. Vieira has several scientific papers and more than 12 years of experience in the field of thin films and devices. Her research activities have been mainly related to the transient analysis and characterization of the transport properties of the semiconductor materials and device characterization.

Isabel Rodrigues was born in Olhão (Portugal) in 1959. She received a university degree in Physics from the Faculty of Sciences of the University of Lisbon, Portugal (1986), and a Master's degree in Applied Mathematics from the Institute of Economics and Management of the 
Technical University of Lisbon, Portugal (1997). At present she teaches Mathematical Analysis in the Electronics, Telecommunications and Computer Department on the Engineering Institute of Lisbon (ISEL-DEETC) and, simultaneously she's doing her PhD in Sensors, Micro and Optoelectronics. Her research interests are related to numerical analysis and simulation of micro and optoelectronic devices.

Rodrigo Ferrão de Paiva Martins was born in New Lisbon, Angola in 1951. He graduated in Electronics Engineering by the University of Luanda in 1974. In 1977, received the MSc in Amorphous Semiconductors Technology by the University of Dundee, Scotland. In 1982, he received the $\mathrm{PhD}$ in Energy Conversion and Semiconducting Materials by the New University of Lisbon. In 1986, he became Associated Professor in the Faculty of Sciences and Technology of the New University of Lisbon and receiving the aggregation by the same institution in 1988 in Semiconductors and Microelectronics. Currently he is Full Professor in FCT/UNL and president of the Material Science Department. Dr. R. Martins has several scientific papers to his credit; was a member of several scientific committees and coordinator of several European projects over the last few years. His main scientific interests are in the field of optoelectronic materials, amorphous semiconductor technology, and applications. 